\title{
Repetitive DNA sequences accelerate molecular cytogenetic research in plants with small chromosomes
}

\author{
Agus Budi Setiawan ${ }^{1, *}$, Ari Wibowo ${ }^{2}$, Chee How $\mathrm{Teo}^{3}$, and Shinji Kikuchi ${ }^{4}$, Takato Koba ${ }^{4}$ \\ ${ }^{1}$ Laboratory of Genetics and Plant Breeding, Faculty of Agriculture, Universitas Gadjah Mada, Jalan Flora Bulaksumur, Yogyakarta 55281, \\ Indonesia \\ ${ }^{2}$ Indonesian Coffee and Cacao Research Institute, Jalan PB. Sudirman No.90, Jember 68175, Indonesia \\ ${ }^{3}$ Center for Research in Biotechnology for Agriculture, University of Malaya, Kuala Lumpur 50603, Malaysia \\ ${ }^{4}$ Laboratory of Genetics and Plant Breeding, Graduate School of Horticulture, Chiba University, Matsudo, Chiba 271-8510, Japan \\ ${ }^{*}$ Corresponding author: setiawanab@ugm.ac.id
}

SUBMITTED 9 July 2019 REVISED 21 August 2019 ACCEPTED 29 November 2019

\begin{abstract}
Repetitive DNA sequences are highly abundant in plant genomes and are favorable probes for chromosome identification in plants. However, it is difficult to conduct studies on the details of metaphase chromosome structures in plants with small chromosomes due to their highly condensed status. Therefore, identification of homologous chromosomes for karyotyping and analyzing chromosome structures is a challenging issue for cytogeneticists without specific probes and precise chromosome stages. In this study, five repetitive DNA probes, i.e., 5S and 45S ribosomal DNAs (rDNAs), melon centromeric sequence (Cmcent), cucumber subtelomeric sequence (Type I), and microsatellite (CT) 10 repeats, were used to identify primary constrictions and homologous chromosomes for karyotyping. Four and two loci of 45S rDNA were respectively observed on metaphase and pachytene chromosomes of Abelia $\times$ grandiflora. Cmcent was detected on both primary constrictions of melon pachytene and metaphase chromosomes. Furthermore, one pair of $5 \mathrm{~S}$ rDNA signals were hybridized on melon metaphase chromosomes. Eight and two loci of 45S and 5S rDNA were respectively detected on cucumber chromosomes. Type I and $(\mathrm{CT})_{10}$ probes were specifically hybridized on subtelomeric and interstitial regions on the chromosomes, respectively. These results suggest that repetitive DNA sequences are versatile probes for chromosome identification in plants with small chromosomes, particularly for karyotyping analyses.
\end{abstract}

KEYWORDS homologous chromosomes; microsatellite repeats; pachytene chromosomes; precise karyotyping; repetitive DNAs

\section{Introduction}

The major proportion of plant genomes is composed of repetitive DNA sequences (Biscotti et al. 2015). These repetitive sequences evolve more rapidly than coding sequences, and they are useful molecular markers for analyzing genetic diversity and studying genome evolution (Kalendar and Schulman 2006). They have been used as cytogenetic probes to investigate the chromosomal organization in plants (Cuadrado et al. 2008), karyotyping markers (Han et al. 2008), and in cytogenetic comparative analysis among closely related species (Zhang et al. 2016). Their chromosomal locations are species-specific at centromeric (Koo et al. 2010), pericentromeric, or subtelomeric regions (Han et al. 2008), or they are found dispersed within chromosome regions (Kubis et al. 1998).

Genome sizes of plants have a strong correlation with chromosome sizes due to the amount of repetitive DNA sequences (Kuznetsova et al. 2017). Consequently, small and large chromosome sizes in plants can affect the mor- phology and visual features of the chromosomes, such as the locations of primary and secondary constrictions, and heterochromatic and euchromatic regions on their metaphase mitotic chromosomes. Chromosome biologists are prefer to use large chromosomes as their model species. However, it is difficult to carry out precise karyotyping in plants with small chromosomes even when well-spread metaphase chromosomes were used due to poor stainability and their highly condensed status (Setiawan et al. 2018c). Therefore, homologous chromosome identification and chromosome structure analysis have been challenging for cytogeneticists without using specific probes and specific chromosome stages such as meiotic pachytene or mitotic prometaphase chromosomes.

Repetitive DNA sequences are classified into two major groups, namely, tandem repeats (micro-, mini-, and satellite DNAs) and dispersed repeats, i.e., transposable elements (DNA transposons and retrotransposons) (Kubis et al. 1998). The 45S rDNA is conserved in the cells of eukaryotic organisms, arranged in the tandem repeat arrays, 
located at the nucleolar organizing region, and encodes 18S, 5.8S, and 25S ribosomal RNAs (Ganal and Hemleben 1986). Furthermore, both microsatellite and satellite repeats are often the primary DNA components in the centromeres and subtelomeric regions, which play important roles in cytogenetic studies (Cuadrado et al. 2008). All these probes have been used as necessary DNA sequences for chromosome identification in several plant species, particularly in identifying homologous and homologous chromosomes (Mendes et al. 2011; Wibowo et al. 2018).

Fluorescence in situ hybridization (FISH) is a useful tool in plant cytogenetic studies and has been used to study the structure, mutation, and evolution of not only individual chromosomes but also entire genomes (Jiang 2019). FISH can be used for physical mapping of any DNA sequences to the chromosomes, the identification, and characterization of individual chromosomes, and the identification of chromosomal rearrangements in the genomes (Schwarzacher and Heslop-Harrison 2000). Since the issue of condensation of metaphase chromosomes in plants with small chromosomes often leads to difficulties in determining the positions of the primary and secondary constrictions and other specific chromosome markers on the chromosomes, probes with specific sequences would be indispensable to overcome such difficulties in cytogenetic studies. In this study, we demonstrate that some specific DNA repeats can be used for the precise identification of homologous chromosomes in facilitating karyotype analyses, particularly in plants with small chromosome sizes.

\section{Materials and Methods}

\subsection{Plant materials}

Abelia $\times$ grandiflora, a hybrid plant between Abelia chinensis and Abelia uniflora; one accession of melon $(\mathrm{Cu}-$ cumis melo L. subsp. agrestis var. conomon), "P90”; and two cucumber cultivars (Cucumis sativus L.), "Power F1 Hybrid" and "Tashu-bashi kyuri" (TBK), were used in this study. The plants were grown and maintained at the Graduate School of Horticulture, Chiba University, Matsudo, Japan.

\subsection{Preparation of meiotic and mitotic chromosomes}

Melon and cucumber seeds were germinated on a moistened filter paper in petri dishes in a growth chamber at $25^{\circ} \mathrm{C}$. The main root tips $(0.5-1 \mathrm{~cm})$ were cut, and the germinated seeds were transplanted into potting trays and maintained in a greenhouse. Before flowering, melon and Abelia $\times$ grandiflora flower buds $(1-1.5 \mathrm{~mm})$ were collected for observing their meiotic cells. Both root tips and flower buds were pretreated with modified Carnoy's solution II containing 6:3:1 (v/v) ethanol: acetic acid: chloroform for 3-4 $\mathrm{h}$ at room temperature (RT) and fixed in C3:1 for 5 days at $4^{\circ} \mathrm{C}$ (Setiawan et al. 2018c). The flower buds were washed in $1 \mathrm{ml}$ of an enzyme buffer $(40 \mathrm{ml}$ of 100 $\mathrm{mM}$ citric acid $+60 \mathrm{ml}$ of $100 \mathrm{mM}$ sodium citrate, $\mathrm{pH} 4.8$ ) for $10 \mathrm{~min}$. The anthers were dissected using forceps under a stereomicroscope. Both root tips and anthers were macerated in $15 \mu \mathrm{l}$ of an enzyme mixture containing $4 \% \mathrm{Cel}-$ lulase Onozuka RS (Yakult), 2\% pectinase (Sigma), and $1 \%$ pectolyase Y-23 (Kyowa Chemical, Osaka, Japan) at $37^{\circ} \mathrm{C}$ for one $\mathrm{h}$. The root tips were processed using the squash technique or flame-dried over an alcohol flame for a few seconds and kept at $-80^{\circ} \mathrm{C}$ for two days, and then the cover slips were removed. The anthers were treated using the ADI method as described by Setiawan et al. (2018c). All slides were kept at RT before conducting FISH.

\subsection{Probe preparation and FISH analysis}

Wheat 45S ribosomal DNA (rDNA; pTa71) (Gerlach and Bedbrook 1979) and melon centromere satellite DNA (Cmcent) (Koo et al. 2010), microsatellite repeat (CT) ${ }_{10}$, Type I (subtelomere-specific 182-bp repeat) (Han et al. 2008), and 5S rDNA were used as probes. 5S rDNA was amplified from melon and cucumber genomic DNAs by polymerase chain reaction (PCR) using the coding regions of 5S rDNA as the primers (Fukui et al. 1994). PCR products with an expected size of 301 bp were cloned into pGEM-T-Easy Vector (Promega) according to the manufacturer's instruction. The Cmcent and 5S rDNA of cucumber were labeled with biotin-nick translation mix (Roche), while Type I, 45S rDNA, and 5S rDNA of melon were labeled with the dig-nick translation mix (Roche). Microsatellite repeat $(\mathrm{CT})_{10}$ was labeled using the end-labeling technique with terminal deoxynucleotidyl transferase in accordance with the manufacturer's instruction (Thermo Scientific) incorporated with biotin-16-dUTP (Roche). FISH analysis was performed according to the method described by (Setiawan et al. 2018c), consisting of chromosome slides pretreated with RNAse A (Qiagen) and pepsin (Sigma), followed by refixation with $1 \%$ paraformaldehyde, hybridization with a labeled DNA probe on a DNA template, including denaturation at $80^{\circ} \mathrm{C}$ for $2 \mathrm{~min}$, and detection of digoxigenin and biotin-labeled probes using anti-digoxigenin rhodamine (Roche) and biotinylated streptavidin-FITC (Vector Laboratories), respectively.

\subsection{Image analysis}

The slides were counterstained with 4',6-diamidino-2phenylindole (DAPI) in a VectaShield antifade solution (Vector Laboratories) before observing under a fluorescence microscope (Olympus BX53) equipped with a cooled CCD camera (Photometrics CoolSNAP MYO). The FISH images were processed by Metamorph, Metavue imaging series version 7.8, and edited with Adobe Photoshop CS 6. The ideogram was constructed using CHIAS IV (Kato et al. 2009). 


\section{Results}

\subsection{Conservation of $45 S$ rDNA on meiotic chromo- somes of Abelia $\times$ grandiflora}

Abelia $\times$ grandiflora has white flowers and small metaphase chromosomes (Figure 1a, d). However, it was difficult to observe the detailed structures of the metaphase chromosomes due to their highly condensed status (Figure 1d). In this study, we observed that Abelia $\times$ grandiflora had 32 chromosomes, and the $45 \mathrm{~S}$ rDNA was successfully hybridized on the interphase, metaphase, and pachytene chromosomes (Figure 1b-d). Two pairs of 45S rDNA signals were observed on the interphase and metaphase chromosomes (Figure 1c-d). One pair of $45 \mathrm{~S}$ rDNA signals were also detected in pachytene cells. The 45S rDNA has a pair of strong and weak signals located at short arms. These results were similar to those of our previous study conducted by Setiawan et al. (2018c). Therefore, these results suggest the conservation of 45S rDNA in Abelia $\times$ grandiflora and that this probe can be used for the identification of homologous chromosomes. Furthermore, because of the issue of condensation in metaphase, pachytene chromosomes were used to investigate the structures of the chromosomes in more detail. The primary constriction of Abelia $\times$ grandiflora was clearly observed on pachytene chromosomes (Figure 1b). Moreover, heterochromatic and euchromatic regions of the chromosomes in the pachytene stage were easily distinguished compared with those of the metaphase stage cells.

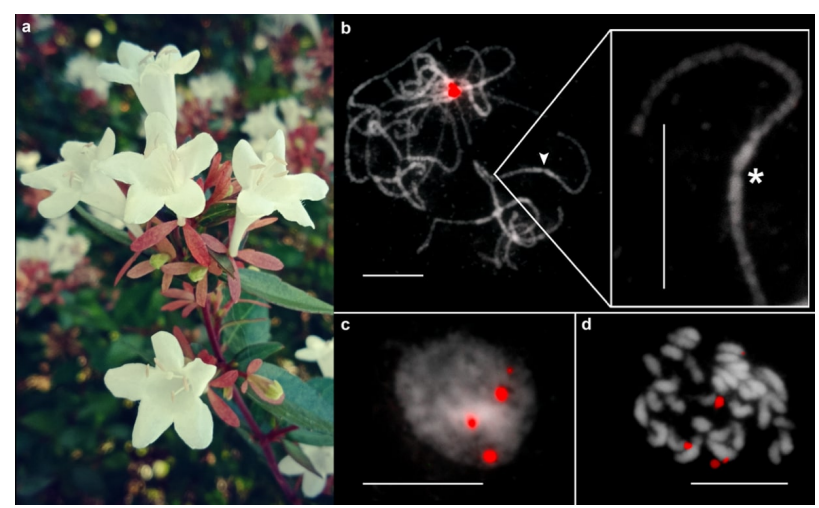

FIGURE 1 Physical mapping of $45 \mathrm{~S}$ rDNA on the meiotic chromosomes of Abelia $x$ grandiflora. (a) The flower morphology of Abelia $x$ grandiflora. FISH detection of $45 \mathrm{~S}$ rDNA on (b) pachytene, (c) interphase, and (d) metaphase chromosomes of Abelia $x$ grandiflora. Red signals depict $45 \mathrm{~S}$ rDNA labeled with digoxigenin. Arrowhead and asterisk depict primary constrictions of Abelia $\mathrm{x}$ grandiflora. Scale bars $=10 \mu \mathrm{m}$.

\subsection{Utilization of DNA repeats for the identification of melon chromosomes}

Cmcent repeats were exclusively hybridized for the detection of the centromeric regions of melon chromosomes (Figure 2a-c). A total of 12 signals of Cmcent were detected in the primary constriction of pachytene chromosomes (Figure 2a). Also, 12 pairs of Cmcent signals were observed in the metaphase and prometaphase chromosomes (Figure $2 \mathrm{~b}-\mathrm{c}$ ). Furthermore, one pair of homologous chromosomes were identified to have 5S rDNA locations on the long arms (Figure 2c). Our results were similar to those of previous studies conducted by Koo et al. (2010), Setiawan et al. (2018c), and Setiawan et al. (2018b). Hence, these results indicate that the application of some DNA repeats is effective for the identification of melon chromosomes.

\subsection{Repetitive DNA sequences promote chromosome identification and karyotyping in cucumber}

A detailed identification of homologous chromosomes using repetitive DNA sequences for facilitating precise karyotyping was conducted in cucumber. The 45S rDNA was successfully hybridized on the metaphase chromosomes of Power F1 Hybrid (Figure 3a). Three pairs of strong and one pair of weak 45S rDNA signals were detected in that cultivar (Fig. 3c). Furthermore, one pair of 5S rDNA signals were detected and located in the regions of the short arms (Figure 3a). These results suggest that 10 of 14 chromosomes of cucumber can be precisely identified using these probe DNAs. The number of $45 \mathrm{~S}$ rDNA signals depends on the cultivars, varying from 8 to 10 signals (Wibowo et al. 2018). We then constructed an ideogram based on the condensation patterns and the hybridization signals of $5 \mathrm{~S}$ and $45 \mathrm{~S}$ rDNAs (Figure 3d). We identified the homologous chromosomes of cucumber and constructed an integrated ideogram of cucumber chromosomes display-
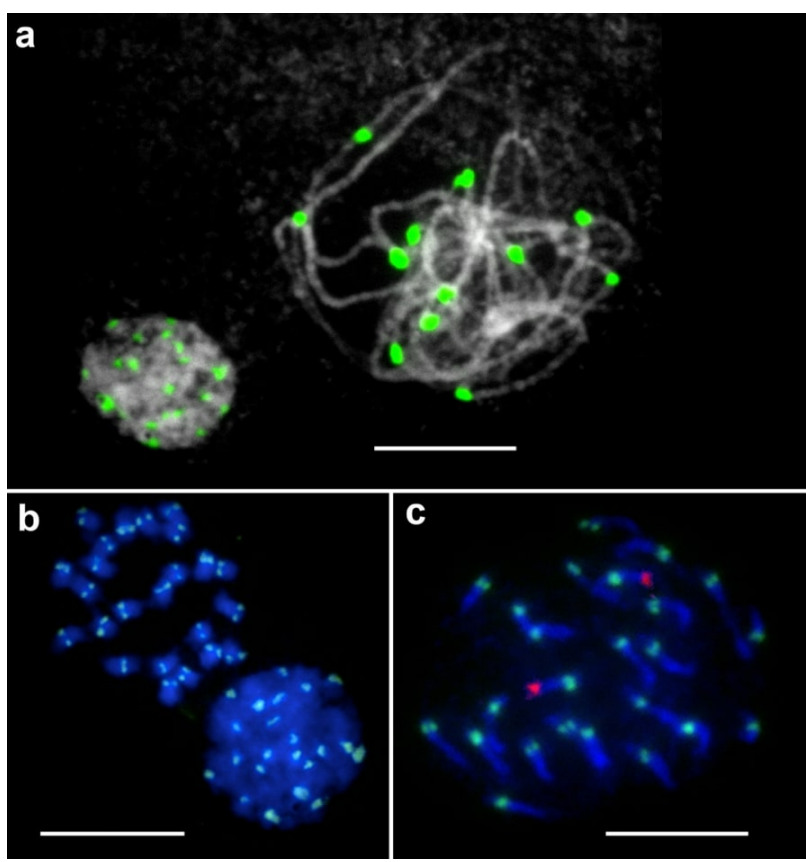

FIGURE 2 Physical mapping of centromeric repeats (Cmcent) and $5 \mathrm{~S}$ rDNA on meiotic and mitotic chromosomes of melon. FISH detection of Cmcent on (a) interphase, pachytene and (b) metaphase chromosomes. 5S rDNA and Cmcent were hybridized on prometaphase chromosomes. Green signals depict Cmcent labeled with biotin. Red signals depict 5S rDNA labeled with digoxigenin. Scale bars $=10 \mu \mathrm{m}$. 

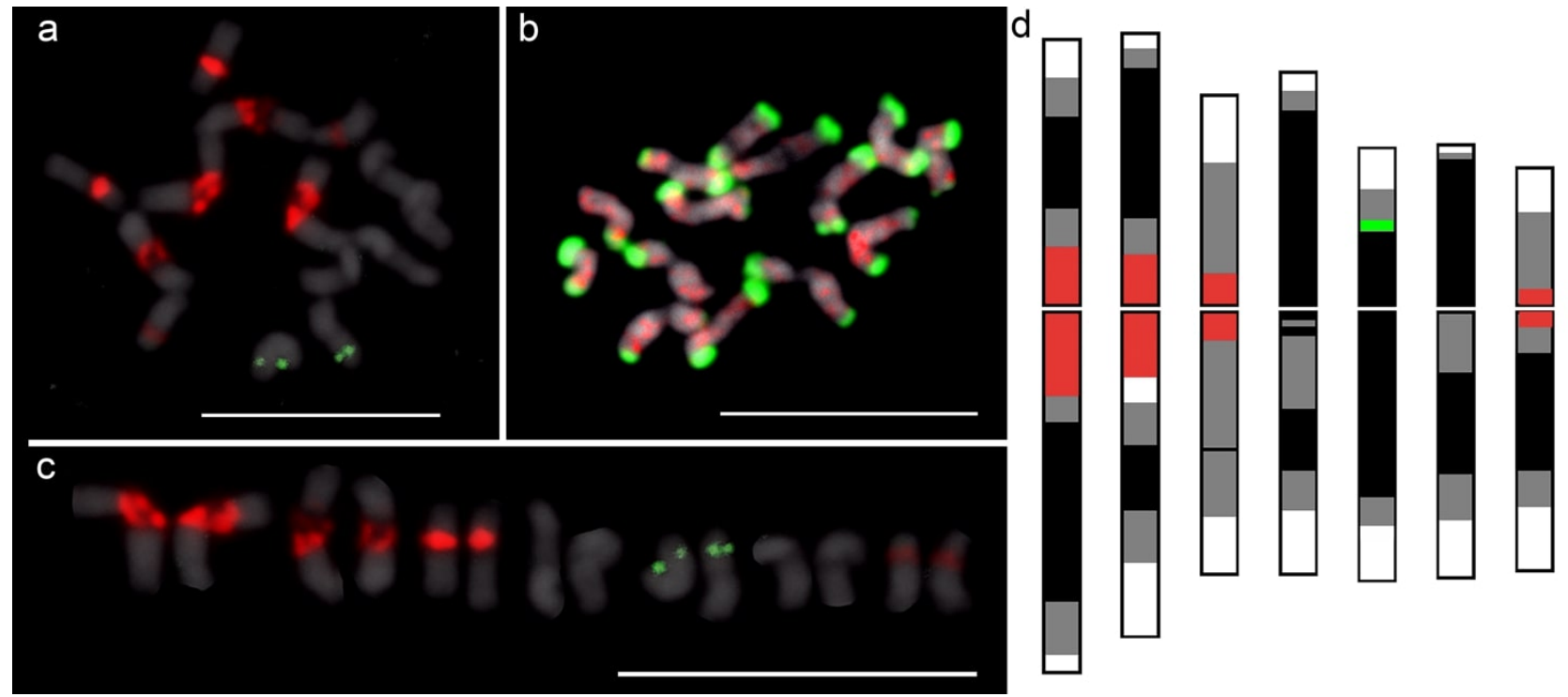

FIGURE 3 Physical mapping of sub-telomeric repeats, microsatellite (CT) $10,5 \mathrm{~S}$ and $45 \mathrm{~S}$ rDNA on mitotic chromosomes of cucumber (a) FISH detection of $5 \mathrm{~S}$ and $45 \mathrm{~S}$ rDNA on prometaphase chromosomes of 'Power F1 Hybrid'; (b) Sub-telomeric repeat (Type I) and (CT)10 hybridized on prometaphase chromosomes of 'TBK'. (c) Homologous prometaphase chromosomes identified in accordance with $5 \mathrm{~S}$ and $45 \mathrm{~S}$ rDNA signals; (d) FISH ideogram of cucumber using prometaphase chromosomes of 'Power F1 Hybrid' based on condensation patterns, 5S (green) and 45S rDNA (red) hybridization signals. Red and green signals in (a) and (c) depict 45S and 5S rDNAs labeled with digoxigenin and biotin, respectively. Red and green signals in (b) depict (CT) 10 and Type I sequences labeled with biotin and digoxigenin, respectively. Black and grey regions in (d) depict heterochromatic and euchromatic regions. Scale bars $=10 \mu \mathrm{m}$.

ing the positions and fluorescence intensities of these DNA repeat signals. Therefore, the $5 \mathrm{~S}$ and $45 \mathrm{~S}$ rDNAs are versatile DNA repeats for chromosome identification in cucumber, particularly for karyotyping analyses. On the other hand, Type I and (CT) 10 repeats were detected on the metaphase chromosomes of the TBK cultivar (Figure 3b). Type I repeats were specifically located on the subtelomeric region of the chromosomes, whereas $(\mathrm{CT})_{10}$ repeats were dispersed within the chromosome arms. Type I repeats could be used to identify the two pairs of chromosomes that did not have the 5S and 45S rDNA signals. Therefore, we found that all somatic chromosomes of cucumber could be distinctly identified by FISH using only Type I, 5S, and 45S rDNA probes.

\section{Discussion}

Repetitive DNA sequences are highly abundant in plant genomes, comprising up to $85 \%$ of the genome in some plant species (Schnable et al. 2009). The melon genome is composed of $42 \%$ of repetitive DNA sequences (GarciaMas et al. 2012). Cucumber genome size is $367 \mathrm{Mb}$ and contains a large number of repetitive sequences (Huang et al. 2009). Repetitive sequences are mostly located at the centromeric, subtelomeric, and nucleolar organizing regions where rDNA sequences have been discovered (Setiawan et al. 2018b; Wibowo et al. 2018). The genomic organization of plant species has been drastically affected by the distribution and copy numbers of various repetitive DNA sequences by forming constitutive heterochromatin (Markova and Vyskot 2010). Their abundance, distribution throughout their genomes, and specific chromosomal localization make them useful as probes for chromosome identification and cytogenetic studies in plants. On the other hand, it is difficult to determine the detailed chromosome structures such as the primary and secondary constrictions in plants with small chromosome sizes when we use metaphase chromosomes. Therefore, we conducted physical mapping of several DNA repeats such as $5 \mathrm{~S}$ and 45S rDNA, centromeric repeats (Cmcent), subtelomeric repeats (Type I), and microsatellite repeats (CT) $)_{10}$ on some plant species.

Satellite DNA is one of the repetitive sequences in plant genomes. Heterochromatic regions are characterized as those with the accumulation of satellite DNAs and favorable sites for centromeres (Han et al. 2008). Therefore, centromeric satellite repeats can be used for identifying the locations of primary constrictions not only for large chromosome models, e.g., onion and wheat, but also for small chromosome models, e.g., Cucumis family, Oryza sativa L., and Abelia $\times$ grandiflora, in which it is difficult to detect the centromeric regions by ordinary chromosome staining. Therefore, centromeric repeats (Cmcent) are useful for determining the positions of the centromeres and the chromosome types according to the relative arm lengths, such as metacentric, submetacentric, telocentric, or acrocentric chromosomes. In this study, Cmcent repeats were hybridized to all primary constrictions of melon chromosomes (Figure 2). These results suggest that repetitive DNAs are significant tools that can be used for karyotyping and chromosome identification.

The genome of cucumber contains a large number of rDNA sequences. It has been estimated that $30 \%$ of unassembled regions of the genome are likely to be het- 
erochromatic satellite or rDNA sequences (Huang et al. 2009). The sequences of ribosomal DNAs, i.e., 5S and $45 \mathrm{~S}$ rDNAs, are conserved in a variety of plant species. However, the number of their loci depends on the species and cultivars. Cucumber has 810 loci of the 45S rDNA (Wibowo et al. 2018), whereas melon has four loci (Setiawan et al. 2018b). The number of 5S rDNA loci in the genus Cucumis of diploid species was conserved with two loci (Setiawan et al. 2018b). The variation in the number of 45S rDNA loci in cucumber is an advantage so that this probe can be used for cytogenetic research. On the other hand, subtelomeric repeats (Type I) could also resolve the problem of identifying the chromosome pairs that could not be detected using $5 \mathrm{~S}$ or $45 \mathrm{~S}$ rDNA probes. Therefore, in this study, all the somatic chromosomes of Power F1 Hybrid could be distinctly discriminated by FISH analysis using only Type I, 5S, and 45S rDNA probes.

A recent development in the FISH technique is the use of synthetic oligonucleotide probes that can be designed from simple sequence repeats or satellite repeats and/or single-copy DNA sequences (Jiang 2019). A synthetic oligonucleotide probe designed from a species can probably be used for application in closely related species. Repetitive DNA probes designed from the cucumber genome were found to exhibit good signals on melon chromosomes and vice versa (Setiawan 2018a). Therefore, the repetitive sequences found in some cultivated plants or model plants can also be used for any related species that are native in tropical areas for the characterization of their karyotypes and physical mapping of the DNA sequences.

\section{Conclusions}

Repetitive DNA sequences are versatile probes for chromosome identification and, particularly, facilitating karyotyping in plants with small chromosomes. Centromeric satellite repeats (Cmcent) were specifically hybridized onto the primary constrictions of melon chromosomes. Mitotic chromosomes of cucumber could be distinctly discriminated using subtelomeric repeat sequences (Type I) and ribosomal DNAs (5S and 45S rDNAs). The repetitive DNA probes could be useful for chromosome identification in plants with small chromosomes and their closely related species.

\section{Acknowledgments}

The authors are grateful to Prof. Kenji Kato (Okayama University) for providing melon seed and Ms. Henda Harmantia Dewi for valuable corrections of the manuscript.

\section{Authors' contributions}

ABS designed the study, performed the research, analyzed the data, and wrote the manuscript. AW and CHT performed the research and analyzed the data. SK and TK provided the plasmid clones of the probes and reviewed and interpreted the data.

\section{Competing interests}

The authors declare no competing interest.

\section{References}

Biscotti MA, Olmo E, Heslop-Harrison JP. 2015. Repetitive DNA in eukaryotic genomes. Chromosome Res 23(3):415-420. doi:10.1007/s10577-015-9499-z.

Cuadrado A, Cardoso M, Jouve N. 2008. Physical organisation of simple sequence repeats (SSRs) in Triticeae: Structural, functional and evolutionary implications. Cytogenet and Genome Res 120(3-4):210219. doi:10.1159/000121069.

Fukui K, Kamisugi Y, Sakai F. 1994. Physical mapping of 5S rDNA loci by direct-cloned biotinylated probes in barley chromosomes. Genome 37(1):105111. doi:10.1139/g94-013.

Ganal M, Hemleben V. 1986. Comparison of the ribosomal RNA genes in four closely related Cucurbitaceae. Plant Syst Evol 154(1-2):63-77. doi:10.1007/BF00984868.

Garcia-Mas J, Benjak A, Sanseverino W, Bourgeois M, Mir G, Gonzalez VM, Henaff E, Camara F, Cozzuto L, Lowy E, et al. 2012. The genome of melon ( $\mathrm{Cu}$ cumis melo L.). Proc Natl Acad Sci 109(29):1187211877. doi:10.1073/pnas.1205415109.

Gerlach W, Bedbrook J. 1979. Cloning and characterization of ribosomal RNA genes from wheat and barley. Nucleic Acids Res 7(7):1869-1885. doi:10.1093/nar/7.7.1869.

Han YH, Zhang ZH, Liu JH, Lu JY, Huang SW, Jin WW. 2008. Distribution of the tandem repeat sequences and karyotyping in cucumber (Cucumis sativus L.) by fluorescence in situ hybridization. Cytogenet Genome Res 122(1):80-88. doi:10.1159/000151320.

Huang S, Li R, Zhang Z, Li L, Gu X, Fan W, Lucas WJ, Wang X, Xie B, Ni P, et al. 2009. The genome of the cucumber, Cucumis sativus L. Nat Genet 41(12):1275-1281. doi:10.1038/ng.475.

Jiang J. 2019. Fluorescence in situ hybridization in plants: recent developments and future applications. Chromosome Res doi:10.1007/s10577-019-09607-z.

Kalendar R, Schulman AH. 2006. IRAP and REMAP for retrotransposon-based genotyping and fingerprinting. Nat Protoc 1(5):2478-2484. doi:10.1038/nprot.2006.377.

Kato S, Ohmido N, Hara M, Kataoka R, Fukui K. 2009. Image analysis of small plant chromosomes by using an improved system, CHIAS IV. Chromosome Sci 12:43-50.

Koo DH, Nam YW, Choi D, Bang JW, De Jong H, Hur Y. 2010. Molecular cytogenetic mapping of Cucumis 
sativus and C. melo using highly repetitive DNA sequences. Chromosome Res 18(3):325-336.

Kubis SE, Heslop-Harrison JS, Desel C, Schmidt T. 1998. The genomic organisation of non-LTR retrotransposons (LINEs) from three Beta species and five other angiosperms. Plant Mol Biol 36:821.

Kuznetsova MA, Chaban IA, Sheval EV. 2017. Visualization of chromosome condensation in plants with large chromosomes. BMC Plant Biology 17(1):1-12. doi:10.1186/s12870-017-1102-7.

Markova M, Vyskot B. 2010. New horizons of genomic in situ hybridization. Cytogenet Genome Res 126(4):368-375. doi:10.1159/000275796.

Mendes S, Moraes AP, Mirkov TE, Pedrosa-Harand A. 2011. Chromosome homeologies and high variation in heterochromatin distribution between Citrus L. and Poncirus Raf. as evidenced by comparative cytogenetic mapping. Chromosome Res 19(4):521-530.

Schnable PS, Ware D, Fulton RS, Stein JC, Wei F, Pasternak S, Liang C, Zhang J, Fulton L, Graves TA, et al. 2009. The B73 maize genome: Complexity, diversity, and dynamics. Science 326(5956):1112-1115. doi:10.1126/science.1178534.

Schwarzacher T, Heslop-Harrison P. 2000. Practical in situ hyridization. New York: Springer.

Setiawan AB. 2018a. Molecular cytogenetic studies on satellite DNA and retrotransposon in Cucumis species. Ph.D. thesis.

Setiawan AB, Teo CH, Kikuchi S, Sassa H, Kato K, Koba T. 2018b. Cytogenetic variation in Cucumis accessions revealed by fluorescence in situ hybridization using ribosomal RNAs genes as the probes. Chromosome Sci 21:67-73. doi:10.11352/scr.21.67.

Setiawan AB, Teo CH, Kikuchi S, Sassa H, Koba T. 2018c. An improved method for inducing prometaphase chromosomes in plants. Mol Cytogenet doi:10.1186/s13039-018-0380-6.

Wibowo A, Setiawan AB, Purwantoro A, Kikuchi S, Koba T. 2018. Cytological Variation of rRNA Genes and Subtelomeric Repeat Sequences in Indonesian and Japanese Cucumber Accessions. Chromosome Sci 21:81-87. doi:10.11352/scr.21.81.

Zhang ZT, Yang Sq, Li ZA, Zhang Yx, Wang Yz, Cheng Cy, Li J, Chen Jf, Lou Qf. 2016. Comparative chromosomal localization of 45S and 5S rDNAs and implications for genome evolution in Cucumis. Genome 59(7):449-457. 